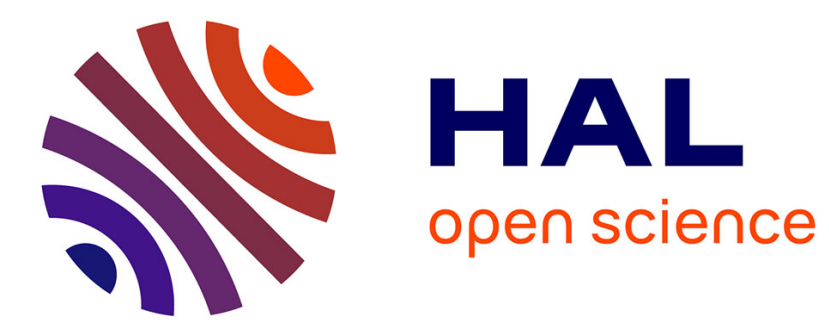

\title{
Equilibrium configurations of compliant tensegrity mechanism based on planar dual-triangles
}

Wanda Zhao, Anatol Pashkevich, Alexandr Klimchik, Damien Chablat

\section{To cite this version:}

Wanda Zhao, Anatol Pashkevich, Alexandr Klimchik, Damien Chablat. Equilibrium configurations of compliant tensegrity mechanism based on planar dual-triangles. ICINCO 2020: International Conference on Informatics in Control, Automation and Robotics, Jul 2020, Paris, France. pp.316-337, 10.1007/978-3-030-92442-3_17. hal-03311969

\section{HAL Id: hal-03311969 \\ https://hal.science/hal-03311969}

Submitted on 2 Aug 2021

HAL is a multi-disciplinary open access archive for the deposit and dissemination of scientific research documents, whether they are published or not. The documents may come from teaching and research institutions in France or abroad, or from public or private research centers.
L'archive ouverte pluridisciplinaire HAL, est destinée au dépôt et à la diffusion de documents scientifiques de niveau recherche, publiés ou non, émanant des établissements d'enseignement et de recherche français ou étrangers, des laboratoires publics ou privés. 


\title{
Equilibrium configurations of compliant tensegrity mechanism based on planar dual-triangles
}

\author{
Wanda Zhao ${ }^{1}$, Anatol Pashkevich ${ }^{1,2}$ Alexandr Klimchik ${ }^{3}$ and Damien Chablat ${ }^{1,4}$ \\ ${ }^{1}$ Laboratoire des Sciences du Numérique de Nantes (LS2N), UMR CNRS 6004, 1 rue de la \\ Noe, 44321 Nantes, France \\ ${ }^{2}$ IMT Atlantique Nantes, 4 rue Alfred-Kastler, Nantes 44307, France \\ ${ }^{3}$ Innopolis University, Universitetskaya St, 1, Innopolis, Tatarstan, 420500, Russia \\ ${ }^{4}$ Centre National de la Recherche Scientifique (CNRS), France
}

\begin{abstract}
The paper deals with the equilibrium configurations and their stability study of a new type of compliant tensegrity mechanism based on dualtriangle structures, which actuated by adjusting elastic connections between the triangle edges. For a single segment of such mechanism, the torque-deflection relation was obtained as a function of control inputs and geometric parameters. It was proved that a single section of the mechanism can has either a single or three equilibrium configurations that can be both stable and unstable. The derived conditions of stability were used to choose control inputs ensuring the mechanism controllability, which was illustrated efficient to give the corresponding control algorithm. The structure composed of two segments in serial was also analyzed and an equivalent serial structure with non-linear virtual springs in the joints was proposed. It was proved that the stiffness of such structure decreases while the external loading increases, which may lead to the buckling phenomenon. Then the control algorithm based on the above principle was carried on, and the obtained results are confirmed by the simulation examples.
\end{abstract}

Keywords: Tensegrity mechanisms; Equilibrium configurations; Stability analysis; Stiffness analysis; Mechanism control.

\section{Introduction}

In designing conventional robotic mechanisms, only rigid parts are usually used for connecting at articulating joints (such as hinges, axles, or bearings), while elastic deformation of links/joints are considered undesirable. However, there are a growing number of applications that require compliant mechanisms that contain passive or active elastic members such as springs or motors, and can gain their motions from the constrained bending of flexible parts. This allows users to increase the flexibilities of devices and achieve complex motions in very constraint environment. Consequently, in the field of robotics, many new kinds of manipulators appeared, with multi degree of freedom, and a large or even infinite degree of kinematic redundancy $[1,2,3,4,5$, 6]. 
Generally, the robotic manipulators can be classified into three types, conventional discrete, serpentine, and continuum robots [7]. The first one is made of traditional rigid components, usually used in industry. The serpentine robots use discrete joints but combine very short rigid links with a large density joints, which produce smooth curves and make the robot similar to a snake or elephant trunk [8]. Different with the serpentine robots, the continuum robots do not contain any rigid links or joints, they are very smooth and soft, bending continuously when working [9].

Both the serpentine and continuum robots received very great attention in the recent years, their highly articulated structures make them well suited for many applications, such as inspection and operation in highly constrained environments [10]. But the pure soft continuum robot cannot provide great output force, and considering at the application field, difficulties of design and analysis, the research of it is not so more as the serpentine robots. Thus, combining rigid and elastic or soft components to make part of robot manipulator becomes a popular and useful practice. The typical earlier hyper-redundant robot designs and implementations can be date to 1970's, [11], which includes a series of plates interconnected by universal joint and elastic control components for pivotable action with respect to one another, through adjusting the elastic control components, it can be pivoted to desired positions resulting in a snake-like movement of the entire arm assembly [12, 13, 14, 15].

Nowadays, a very promising trend in compliant robotics is using a series of similar segments based on varies tensegrity mechanisms, which are assembly of compressive elements and tensile elements (cables or springs) held together in equilibrium [16, 17]. This paper concentrates on the stiffness analysis and equilibrium stability of a new type of compliant tensegrity mechanism composed of two rigid triangle parts, which are connected by a passive joint in the center and two elastic edges on each sides with controllable preload. Some kinds of the tensegrity mechanisms have been already studied carefully in literature [18, 19]. In particular, the cable-driven X-shape tensegrity structures were considered in [20,21], where each section was composed of four fixed-length rigid bars and two springs. For this mechanism, the authors investigated influence on the cable lengths on the mechanism equilibrium configurations, which maybe both stable and unstable. Special attention was paid to the work space and singularities analysis. Another group of related works [19] deals with the mechanism composed of two springs and two length-changeable bars. The authors analyzed the mechanism stiffness using the energy method, and demonstrated that the stiffness of this mechanism always decreases when it is subjected to external loads with the actuators locked, which may lead to "buckling". Some other research in this area [22] focus on the three-spring mechanisms, for which the equilibrium configurations stability and singularity were analysed. Using these results the authors obtained conditions under which the mechanism can work continuously, without the "buckling" or "jump" phenomenon. There are also some research studying a four-legged parallel platform [23], which is based on the compliant tensegrity mechanisms. Here, each leg consists of a piston and a spring in series, which allows the platform to achieve in the desired position and orientation. The authors investigated the loaded equilibrium configurations and numerically computed the platform stiffness. However, the tensegrity mechanism based on dual-triangles were not studied in robotic literature yet. 
This paper focuses on the stiffness analysis of a new tensegrity mechanism, which is based on rigid dual-triangles connected by a passive joint that is actuated by adjusting elastic connections between the remaining triangle edges. This structure is proved to be very promising for designing of multi-section series chain possessing very high flexibility [24]. The loaded and unloaded stiffness analysis of two-segment mechanism were carried out in detail. The results provide a good base of the study of the multi-segment manipulators in the future work. While different from our previous research, here we concentrate on the equilibriums computing, the stability analysis and the selection of the mechanism geometric parameters, which are both for symmetrical and nonsymmetrical control inputs of this mechanism. Then one control algorism based on the equilibrium condition for this mechanism was presented in this paper, which allows to define the suitable control inputs for the elastic parts of this mechanism and get the desired equilibrium configurations. The simulation for both loaded and unloaded mode were carried on, the results demonstrated that this algorithm can maintain the desired stable equilibrium configuration, and avoid the negative equivalent rotational stiffness causing unfeasibility of the desired configuration.

\section{Analysis of A Single Segment Mechanism}

\subsection{Geometrical Model and Static Equilibrium Equation}
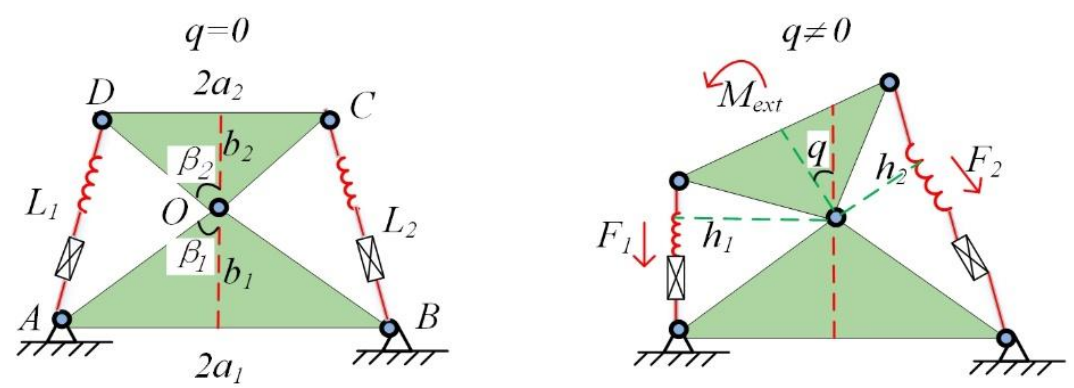

Fig. 1. Geometry of a single segment mechanism.

Let us consider first a 1-d.o.f. segment of the total flexible structure to be studied, which consists of two rigid triangles connected by a passive joint whose rotation is constrained by two linear springs as shown in Fig. 1. It is assumed that the mechanism geometry is described by the triangle parameters $\left(a_{1}, b_{1}\right)$ and $\left(a_{2}, b_{2}\right)$, and the mechanism shape is defined by the angle that can be adjusted by means of two control inputs influencing on the spring lengths $L_{1}$ and $L_{2}$. Let us denote the spring lengths in the non-stress state as $L_{1}^{0}$ and $L_{2}^{0}$, and the springs stiffness coefficients $k_{1}$ and $k_{2}$. 
(a) monotonic case: one equilibrium

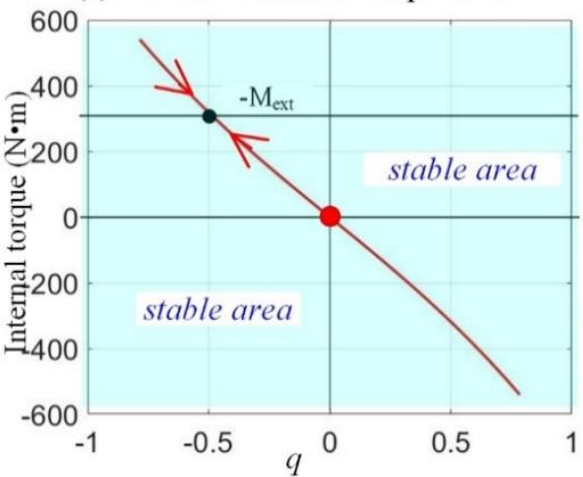

(b) non-monotonic case: three equilibriums

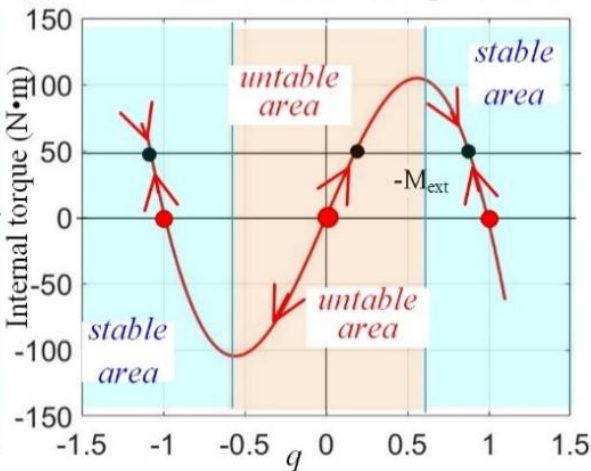

Fig. 2. The torque-angle curves and static equilibriums for $L_{1}^{0}=L_{2}^{0}\left(q_{0}=0\right)$.

To find the mechanism configuration angle $q$ corresponding to given control inputs $L_{1}^{0}$ and $L_{2}^{0}$, let us derive first the static equilibrium equation. Here, the forces $F_{1}$, $F_{2}$ generated by the springs can be obtained from Hook's law as follows.

$$
F_{1}=k_{1}\left(L_{1}-L_{1}^{0}\right) ; \quad F_{2}=k_{2}\left(L_{2}-L_{2}^{0}\right)
$$

where $L_{1}$ and $L_{2}$ are the spring lengths $|A D|,|B C|$ corresponding to the current value of the angle $q$. These values can be computed from the triangles $\triangle A O D$ and $\triangle B O C$ using the formulas

$$
\begin{aligned}
& L_{1}\left(\theta_{1}\right)=\sqrt{c_{1}^{2}+c_{2}^{2}+2 c_{1} c_{2} \cos \left(\theta_{1}\right)} \\
& L_{2}\left(\theta_{2}\right)=\sqrt{c_{1}^{2}+c_{2}^{2}+2 c_{1} c_{2} \cos \left(\theta_{2}\right)}
\end{aligned}
$$

where $c_{1}=\sqrt{a_{1}^{2}+b_{1}^{2}}, c_{2}=\sqrt{a_{2}^{2}+b_{2}^{2}}$ and the angles $\theta_{1}, \theta_{2}$ are expressed via the mechanism parameters as follows

$$
\theta_{1}=\beta_{12}+q, \theta_{2}=\beta_{12}-q, \beta_{12}=\operatorname{atan}\left(a_{1} / b_{1}\right)+\operatorname{atan}\left(a_{2} / b_{2}\right)
$$

The torques $M_{1}=F_{1} \cdot h_{1}, M_{2}=F_{1} \cdot h_{1}$ created by the forces $F_{1}, F_{2}$ in the passive joint $O$ can be computed using the triangle area relations $L_{1} h_{1}=c_{1} c_{2} \sin \left(\theta_{1}\right)$, $L_{2} h_{2}=c_{1} c_{2} \sin \left(\theta_{2}\right)$ of $\triangle A O D$ and $\triangle B O C$, which yield the following expressions

$$
\begin{aligned}
& M_{1}(q)=+k_{1}\left(1-L_{1}^{0} / L_{1}\left(\theta_{1}\right)\right) c_{1} c_{2} \sin \left(\theta_{1}\right) \\
& M_{2}(q)=-k_{2}\left(1-L_{2}^{0} / L_{2}\left(\theta_{2}\right)\right) c_{1} c_{2} \sin \left(\theta_{2}\right)
\end{aligned}
$$

where the difference in signs is caused by the different direction of the torques generated by the forces $F_{1}, F_{2}$ with respect to the passive joint. 
Further, taking into account the external torque $M_{\text {ext }}$ applied to the moving platform, the static equilibrium equation for the considered mechanism can be written as follows

$$
M_{1}(q)+M_{2}(q)+M_{e x t}=0
$$

Solving this equation we can get the rotation angle $q$ defining the mechanism equilibrium configuration and corresponding to the control inputs $L_{1}^{0}, L_{2}^{0}$ and the external torque $M_{e x t}$ applied to the moving platform. This equation is highly nonlinear and cannot be solved analytically, so it is reasonable to apply the numerical Newton technique, which leads to the iterative scheme

$$
q^{k+1}=q^{k}-\left(M\left(q^{k}\right)+M_{e x t}\right) / M^{\prime}\left(q^{k}\right)
$$

where $M(q)=M_{1}(q)+M_{2}(q)$, and the derivative $M^{\prime}(q)=d M(q) / d q$ can be computed analytically, in order to speed up the computations.

\subsection{Equilibrium Configurations and Their Stability}

Let us now evaluate the stability of the mechanism under consideration, which shows its resistance against the external disturbances. In general, this property highly depends on the equilibrium configuration defined by the angle $q$, which satisfies the equilibrium equation $M(q)+M_{\text {ext }}=0$. As follows from the relevant analysis, the function $M(q)$ can be either monotonic or non-monotonic one, so the single-segment mechanism under study may have multiple stable and unstable equilibriums, which are studied in detail below.

To analyze the mechanism equilibriums, let us consider the torque-angle curves $M(q)=M_{1}(q)+M_{2}(q)$ defined by (3) and presented in Fig. 2 [24]. It is clear that for the monotonic function $M(q)$ with negative derivative ( see Fig. 2a) increase of the external loading $M_{e x t}$ always leads to higher mechanism resistance, so the equilibrium is unique and stable. However, in the non-monotonic case, while increasing the external loading, it is possible to achieve a point where the mechanism does not resist any more and suddenly changes its configuration as shown in Fig. 2b. It is worth mentioning that similar phenomenon can be observed in other mechanism and is known in mechanics as "buckling" [25]. Hence, in the non-monotonic case, there maybe three solutions of the equilibrium equation (two stables and one unstable).

As follows from the above presented figures, the static equilibrium defined by angle $q$ is stable if and only if the corresponding derivative $M^{\prime}(q)$ is negative. However, taking into account possible shapes of the torque-angle curves $M(q)$ that can be either monotonic or two-model one, the considered stability condition can be simplified and reduced to the derivative sign verification at the zero point only, i.e. 


$$
\left.M^{\prime}(q)\right|_{q=0}<0
$$

which is easy to verify in practice. It should be noted that here the derivative $M^{\prime}(q)$ represents the equivalent rotational stiffness of the mechanism for the unloaded configuration with $q=0$.

To express the above derivative $M^{\prime}(q)$ analytically, let us represent the function $M(q)$ in the following way

$$
M(q)=c_{1} c_{2} k_{1}\left(1-\frac{L_{1}^{0}}{L_{1}\left(\theta_{1}\right)}\right) \cdot \sin \theta_{1}-c_{1} c_{2} k_{2}\left(1-\frac{L_{2}^{0}}{L_{2}\left(\theta_{2}\right)}\right) \cdot \sin \theta_{2}
$$

This allows us to express the mechanism stiffness in general case as follows

$$
\begin{aligned}
M^{\prime}(q)= & c_{1} c_{2} k_{1}\left(1-\frac{L_{1}^{0}}{L_{1}\left(\theta_{1}\right)}\right) \cos \theta_{1}+c_{1} c_{2} k_{2}\left(1-\frac{L_{2}^{0}}{L_{2}\left(\theta_{2}\right)}\right) \cos \theta_{2}- \\
& -\frac{c_{1}^{2} c_{2}^{2} k_{1} L_{1}^{0}}{\left(L_{1}\left(\theta_{1}\right)\right)^{3}} \sin ^{2} \theta_{1}-\frac{c_{1}^{2} c_{2}^{2} k_{2} L_{2}^{0}}{\left(L_{2}\left(\theta_{2}\right)\right)^{3}} \sin ^{2} \theta_{2}
\end{aligned}
$$

For the special cases $q=0$ and $q= \pm \beta_{12}$, which are needed for further analysis, the above expression is simplified respectively to

$$
\begin{gathered}
\left.M^{\prime}(q)\right|_{q=0}=-c_{1}^{2} c_{2}^{2}\left(k_{1} L_{1}^{0}+k_{2} L_{2}^{0}\right) \frac{\sin ^{2} \beta_{12}}{\left(L\left(\beta_{12}\right)\right)^{3}}+c_{1} c_{2} \cos \beta_{12}\left(k_{1}+k_{2}-\frac{k_{1} L_{1}^{0}+k_{2} L_{2}^{0}}{L\left(\beta_{12}\right)}\right) \\
\left.M^{\prime}(q)\right|_{q_{0}=\beta_{12}}=c_{1} c_{2} k_{1} \cos 2 \beta_{12}\left(1-\frac{L_{1}^{0}}{L\left(2 \beta_{12}\right)}\right)+ \\
+c_{1} c_{2} k_{2}\left(1-\frac{L_{2}^{0}}{c_{1}+c_{2}}\right)-c_{1}^{2} c_{2}^{2} k_{1} L_{1}^{0} \frac{\sin ^{2} 2 \beta_{12}}{\left(L\left(2 \beta_{12}\right)\right)^{3}}
\end{gathered}
$$

where $\quad L\left(\beta_{12}\right)=\sqrt{c_{1}^{2}+c_{2}^{2}+2 c_{1} c_{2} \cos \beta_{12}}, L\left(2 \beta_{12}\right)=\sqrt{c_{1}^{2}+c_{2}^{2}+2 c_{1} c_{2} \cos 2 \beta_{12}}$.

Here the geometry constrain is taken into account which making sure that triangles $\triangle A O D$ and $\triangle B O C$ (See Fig. 1) exist at the same time.

Let us also consider in detail the symmetrical case, for which $a_{1}=a_{2}, b_{1}=b_{2}$, $c_{1}=c_{2}, k_{1}=k_{2}, L_{1}^{o}=L_{2}^{o}$. In this case, we can omit some indices and present the torque-angle relationship as well as the stiffness expression in the more compact forms:

$$
M(q)=2 c k\left(c \cos \beta_{12} \sin q-L^{0} \cos \frac{\beta_{12}}{2} \sin \frac{q}{2}\right)
$$




$$
M^{\prime}(q)=c k\left(2 c \cos \beta_{12} \cos q-L^{0} \cos \frac{\beta_{12}}{2} \cos \frac{q}{2}\right)
$$

(a)

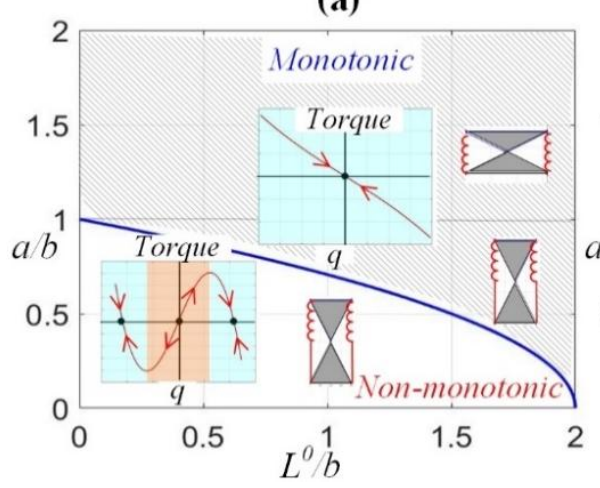

(b)

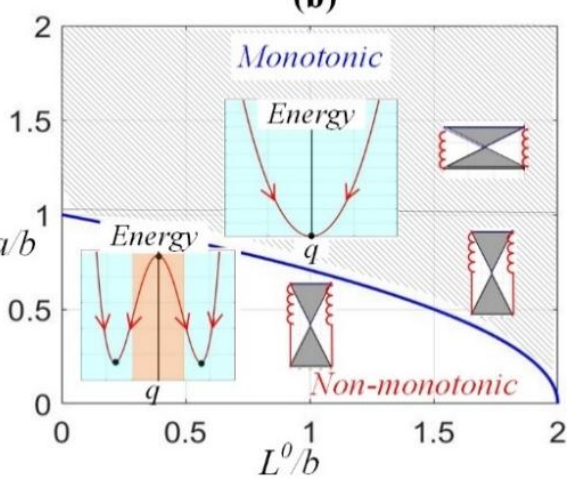

Fig. 3. Monotonic and non-monotonic regions of the parameter plane for $L_{1}^{o}=L_{2}^{o}$

where the control input $L^{o}$ must satisfy the condition $0<L^{o}<2 b$, as follows from the mechanism geometry (Fig. 1). To distinguish the monotonic and non-monotonic cases presented in Fig. 2, it is also necessary to compute the derivative $M^{\prime}(q)$ for the unloaded equilibrium configuration $q=0$, which after simplification can be expressed in the following way

$$
\left.M^{\prime}(q)\right|_{q=0}=k\left(2\left(b^{2}-a^{2}\right)-L^{o} b\right)
$$

The latter allows us to present the condition (6) of torque-angle curve monotonicity as

$$
L^{0} / b>2\left(1-(a / b)^{2}\right)
$$

and separate the parameter plane in two regions, monotonic and non-monotonic ones as shown in Fig. 3. As follows from Fig. 3a, the unloaded equilibrium is always stable if $a>b$. Otherwise, to have stable unloaded equilibrium, the control inputs $L_{1}^{o}=L_{2}^{o}$ should be higher than certain value

$$
L_{i}^{o}>2 b\left[1-(a / b)^{2}\right] ; \quad i=1,2
$$

The monotonic and non-monotonic cases are also illustrated by Fig. 3b, which includes the energy curves $E(q)=\frac{1}{2} \sum_{i=1}^{2} k\left(L_{i}(q)-L^{0}\right)^{2}$ as the function of the rotation angle $q$. As follows from this figure, the energy $E(q)$ has either a single minimum $q=0$ corresponding to a stable equilibrium, or two symmetrical minima 


$$
q_{e}= \pm 2 \arccos \left(\frac{L^{0} b}{2\left(b^{2}-a^{2}\right)}\right)
$$

(a) $q_{\mathrm{e}}<\beta_{12}$

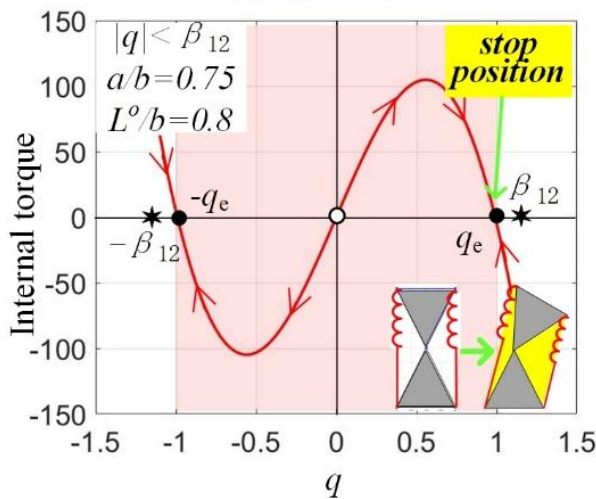

(b) $q_{\mathrm{e}}>\beta_{12}$

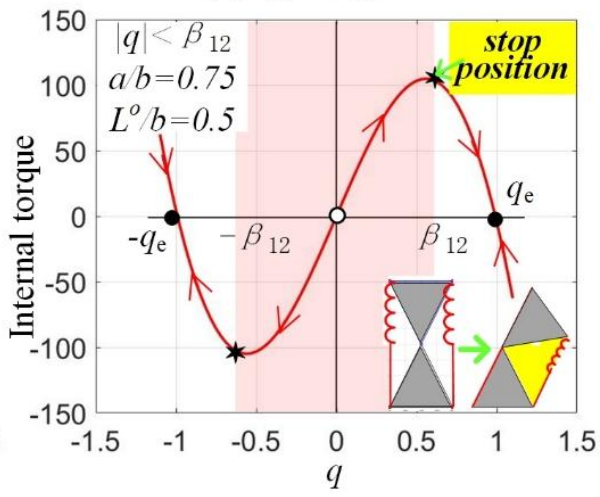

Fig. 4. Location of stable "•" and unstable "o" equilibriums with respect to geometric boundary $\left[-\beta_{12}, \beta_{12}\right]$.

and a local maximum $q=0$ corresponding to two stable equilibriums and one unstable equilibrium.

For the symmetrical case with equal control inputs $L_{1}^{0}=L_{2}^{0}$, let us also compute the torques (7) at the boundary points $q= \pm \beta_{12}$.

$$
\left.M(q)\right|_{q= \pm \beta_{12}}= \pm \frac{2 a b k}{a^{2}+b^{2}}\left(2\left(b^{2}-a^{2}\right)-L^{0} \sqrt{a^{2}+b^{2}}\right)
$$

which allows us to decide if the stable equilibriums in the non-monotonic case are located inside of the interval of feasible rotation angles $q \in\left[-\beta_{12},+\beta_{12}\right]$. It can be proved that the relevant condition can be expressed as (18), and allows user to estimate if the energy minimum is achieved inside or on the border of the feasible region of $q$. A physical interpretation of this non-equality is shown in Fig. 4, where two cases are presented. In the first case, the mechanism is unstable in the desired configuration $q=0$ and jumps to one of two possible stable configurations $q= \pm q_{e}$ that are located inside of mechanical limits.

$$
L^{0}>\frac{2\left(b^{2}-a^{2}\right)}{\sqrt{b^{2}+a^{2}}}
$$

In the second case, the mechanism is also unstable in the equilibrium configuration $q=0$ but it jumps to one of the mechanical limits $q= \pm \beta_{12}$ (because the stable configurations are out of the limits). So, a static error appears in both cases, whose value is 
equal to either $\pm \beta_{12}$ or $\pm q_{e}$. For this reason, it is necessary to avoid in practice the parameters combinations producing non-monotonic torque-angle curves.
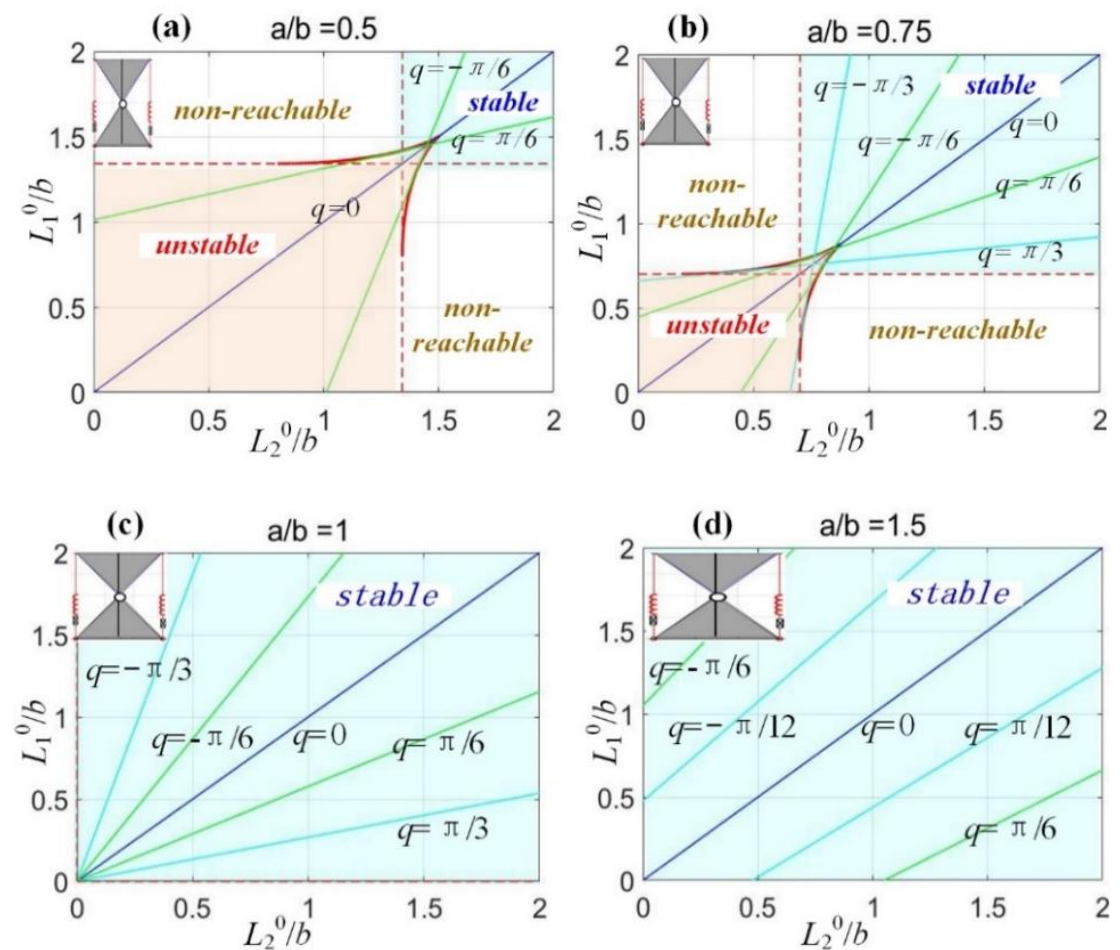

Fig. 5. Regions of equilibrium stability for different inputs $L_{1}^{0}, L_{2}^{0}$.

It is also useful to investigate the case when the control inputs are not equal, i.e. $L_{1}^{0} \neq L_{2}^{0}$, assuming that they produce the desired stable configuration with the output angle $q \neq 0$. In this case, the torque and its derivative can be presented as follows.

$$
\begin{gathered}
M(q)=2 c^{2} k \cos \beta_{12} \sin q-c k\left(L_{1}^{0} \sin \frac{\beta_{12}+q}{2}-L_{2}^{0} \sin \frac{\beta_{12}-q}{2}\right) \\
M^{\prime}(q)=2 k\left(b^{2}-a^{2}\right) \cos q-k\left(\frac{L_{1}^{0}+L_{2}^{0}}{2} b \cos \frac{q}{2}-\frac{L_{1}^{0}-L_{2}^{0}}{2} a \sin \frac{q}{2}\right)
\end{gathered}
$$

where all notations are the same as in the above expressions (7) and (8). It is clear that to implement such control, the desired configuration must correspond to stable equilibrium for which $M(q)=0, M^{\prime}(q)<0$. It is also obvious that for $L_{1}^{0}<L_{2}^{0}$ the equilibrium correspond to positive angle $q>0$, and vice versa. 
It can be proved from the equilibrium equation that the control inputs $L_{1}^{0}, L_{2}^{0}$ insuring the desired output angle $q$ must satisfy the linear relation

$$
L_{1}^{0} \sin \frac{\beta_{12}+q}{2}-L_{2}^{0} \sin \frac{\beta_{12}-q}{2}=2 c \cos \beta_{12} \sin q
$$

which gives infinite set of control variables $\left(L_{1}^{0}, L_{2}^{0}\right)$, which may correspond either to a stable or unstable equilibrium, depending on the derivative $M^{\prime}(q)$. To analyze sign of the derivative, let us consider separately two cases: $a>b$ and $a<b$. In the first case, when $a>b$ and mechanism geometry imposes the constrain $|q|<\pi / 2$, so all three terms of (20) are negative, and the desired equilibrium configuration $q$ is stable. In the second case, when $a<b$ and the angle $q$ maybe out of the range $[-\pi / 2, \pi / 2]$, and the equilibrium maybe unstable. Corresponding separation curves can be found from the conditions $M(q)=0$ and $d M(q) / d q=0$, which yield the following system of linear equations with respect to the control variables $L_{1}^{0}, L_{2}^{0}$.

$$
\begin{gathered}
\left(\sin \frac{\beta_{12}+q}{2}\right) L_{1}^{0}-\left(\sin \frac{\beta_{12}-q}{2}\right) L_{2}^{0}=2 c \cos \beta_{12} \sin q \\
\left(\frac{a}{2} \sin \frac{q}{2}-\frac{b}{2} \cos \frac{q}{2}\right) L_{1}^{0}-\left(\frac{a}{2} \sin \frac{q}{2}+\frac{b}{2} \cos \frac{q}{2}\right) L_{2}^{0}=-2\left(b^{2}-a^{2}\right) \cos q
\end{gathered}
$$

whose solution allows us to present the stability condition in the following form.

$$
\begin{aligned}
& \frac{L_{1}^{0}}{b}>2\left(\frac{b}{a}-\frac{a}{b}\right)\left(\frac{a}{b} \cos ^{3} \frac{q}{2}+\sin ^{3} \frac{q}{2}\right) \\
& \frac{L_{2}^{0}}{b}>2\left(\frac{b}{a}-\frac{a}{b}\right)\left(\frac{a}{b} \cos ^{3} \frac{q}{2}-\sin ^{3} \frac{q}{2}\right)
\end{aligned}
$$

It is worth mentioning that in the case of $q=0$ the above expressions give the stability condition (24).

Hence, to achieve the desired configuration $q$ it is necessary to apply the control inputs $L_{1}^{0}, L_{2}^{0}$ satisfying both the equilibrium condition (22) and the stability conditions (23). Corresponding regions of $L_{1}^{0}, L_{2}^{0}$ are presented in Fig. 5, which clearly shows for which combination of control inputs the desired configuration $q$ can be reached geometrically and it is statically stable. This results are also confirmed by the case study presented in Fig. 6 that demonstrate the monotonic and non-monotonic torque-angle curves for the parameters $\mathrm{a} / \mathrm{b}=0.75$, and control inputs (a) $L_{1}^{0}=0.88 b$, $L_{2}^{0}=0.92 b$, and (b) $L_{1}^{0}=0.75 b, L_{2}^{0}=0.65 b$, which produce the unloaded equilibrium 
configurations with the same angle $q_{0}=\pi / 6$ (but with different stability properties). It should be also noted that here there are only two equilibriums in the non-monotonic case presented in Fig. 6b, while normally three equilibriums exist for equal control inputs as shown in Fig. 2b. The latter is caused by the geometric constrains which eliminate the third equilibrium for non-equal control inputs.

(a) monotonic case: one equilibrium

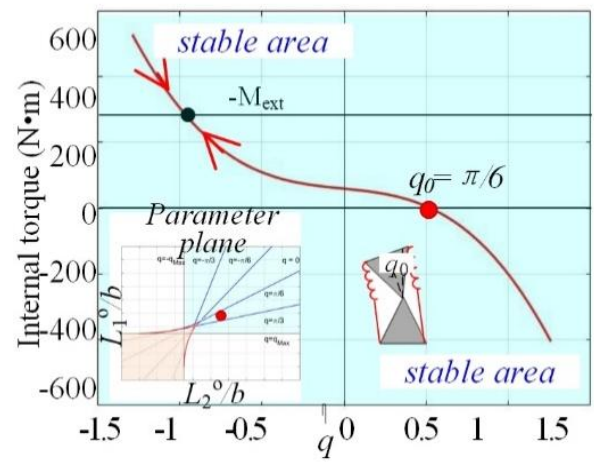

(b) non-monotonic case: three equilibriums

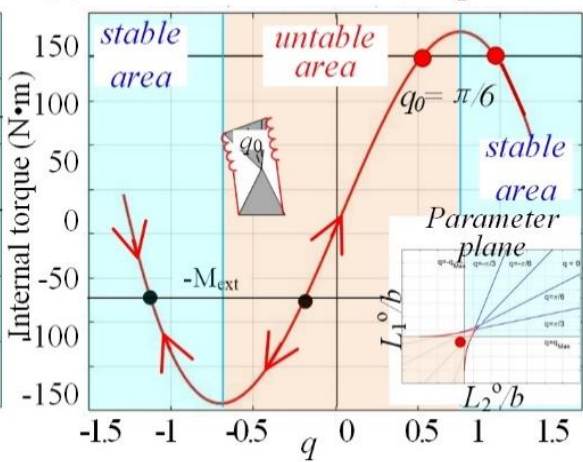

Fig. 6. The torque-angle curves and static equilibriums for $L_{1}^{0} \neq L_{2}^{0}\left(q_{0}=\pi / 6\right)$.

\subsection{Controlling Mechanism Configurations}

As follows from the mechanism structure (see Fig. 1), the desired configuration is defined by single variable $q$ which is adjusted by two control variables $L_{1}^{0}$ and $L_{2}^{0}$. The latter creates redundancy and ambiguity in control inputs selection. To eliminate this difficulty, it is reasonable to define $L_{1}^{0}$ and $L_{2}^{0}$ in a symmetrical way, i.e. as $L_{1}^{0}=L^{0}-\Delta$ and $L_{2}^{0}=L^{0}+\Delta$. This allows us to rewrite (19) as follows

$$
M_{q}=2 k\left[\left(b^{2}-a^{2}\right) \sin (q)-L^{0} b \cdot \sin \left(\frac{q}{2}\right)+\Delta \cdot a \cdot \cos \left(\frac{q}{2}\right)\right]
$$

and present the control law $\Delta(q)$ for the unloaded case $\left(M_{e x t}=0\right)$ in the following way

$$
\Delta(q)=\frac{L^{0} b \sin (q / 2)-\left(b^{2}-a^{2}\right) \sin (q)}{a \cos (q / 2)}
$$

It should be noted that the desired configuration defined by the angle $q$ should always satisfy the geometric constrains

$$
\begin{array}{ll}
|q|<2 \operatorname{atan}(a / b), & a \leq b \\
|q|<\pi-2 \operatorname{atan}(a / b), & a>b
\end{array}
$$



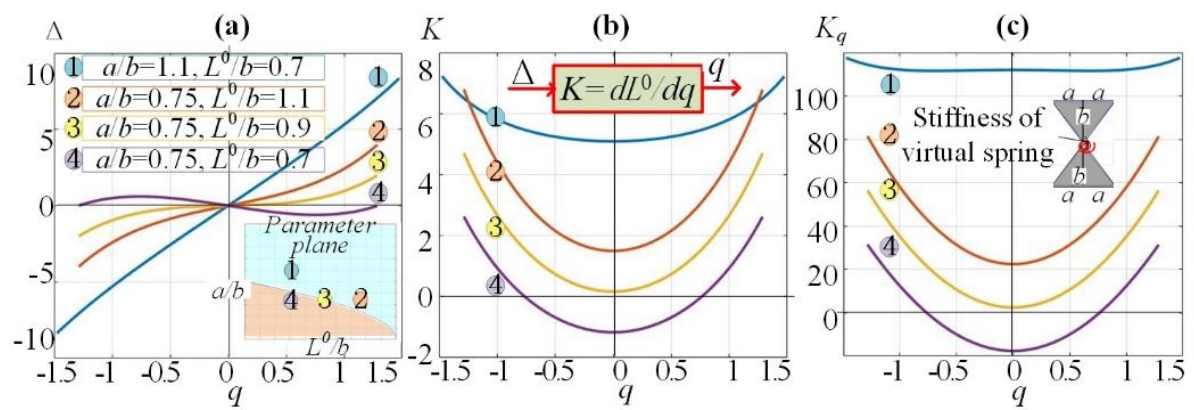

Fig. 7. Relations between the control input $\Delta$, sensitivity coefficient $K$, stiffness coefficient $K_{q}$ and the desired configuration angle $q$ (unloaded case $M_{e x t}=0$ ).
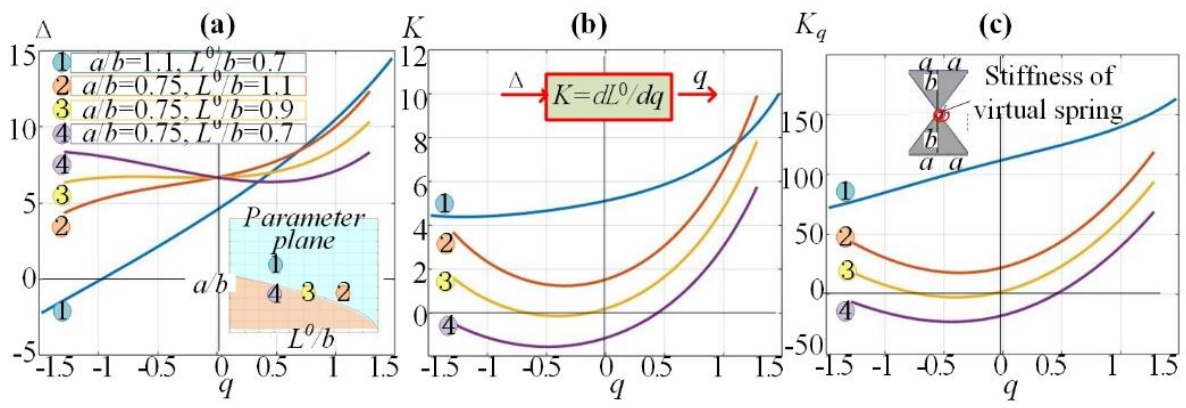

Fig. 8. Relations between the control input $\Delta$, sensitivity coefficient $K$, stiffness coefficient $K_{q}$ and the desired configuration angle $q$ (loaded case $M_{\text {ext }} \neq 0$ ).

that can be easily obtained from Fig. 1. The obtained control law $\Delta(q)$ as well as the corresponding sensitivity and stiffness coefficient are presented in Fig. 7. As follows, from these figures, for the proposed control strategy it is necessary carefully select initial values of control inputs $L_{1}^{0}=L_{2}^{0}$, in order to avoid the negative equivalent rotational stiffness causing instability of the desired configuration of the mechanism.

It is worth mentioning that the above equations were derived assuming that the external loading $M_{e x t}$ is equal to zero. So, in more general case when $M_{e x t} \neq 0$, the control law must be revised, the corresponding sensitivity and stiffness coefficient are presented in Fig. 8. It can be proved that to achieve the desired configuration with the angle $q$ and the external loading $M_{e x t}$, the control input $\Delta$ should be computed using an expression

$$
\Delta\left(q, M_{e x t}\right)=\frac{M_{e x t} / 2 k+L^{0} b \cdot \sin (q / 2)-\left(b^{2}-a^{2}\right) \sin (q)}{a \cdot \cos (q / 2)}
$$

which shows that in the loaded case, the symmetrical configuration with $q_{0}=0$ is achieved by applying a non-zero control input that compensate the external loading. 
However, it is necessary to be careful about selection of the parameter $L^{0}$ which in some cases can cause negative stiffness leading to the buckling phenomenon.

\section{Analysis of A Two-Segment Mechanism}

\subsection{Stiffness Analysis of A Straight Configuration}
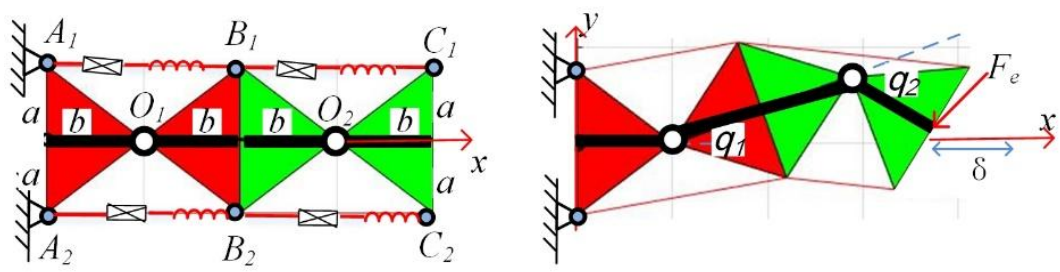

Fig. 9. The two segment mechanism in the "straight" and "non-straight" configurations.

Let us consider first an aggregated mechanism presented in Fig. 9, which is composed of two segments considered in the previous section. It is assumed that the left handside of the mechanism is fixed and the desired configuration corresponds to the "straight" shape with $q_{1}=q_{2}=0$ that is achieved by applying equal control inputs to all segments. Under the influence of the external force $F_{e}$, the end-effector moves from the unloaded equilibrium configuration $\left(x_{0}, y_{0}\right)^{T}=(4 b, 0)^{T}$ to a new equilibrium with the end-effector location $(x, y)^{T}=\left(4 b-\delta_{x}, \delta_{y}\right)^{T}$ corresponding to the nonzero angles $\left(q_{1}, q_{2}\right)$. Let us evaluate the mechanism resistance to the external force $F_{e}$ for this "straight" configuration described by the force-deflection relations $F_{x}\left(\delta_{x}, \delta_{y}\right)$ and $F_{y}\left(\delta_{x}, \delta_{y}\right)$.

It is clear that if the end-effector deflection $\left(\delta_{x}, \delta_{y}\right)$ is given, the configuration angles $\left(q_{1}, q_{2}\right)$ can be computed from the mechanism geometry, using the triangle equations

$$
\begin{aligned}
& b+2 b C_{1}+b C_{12}=4 b-\delta_{x} \\
& 2 b S_{1}+b S_{12}=\delta_{y}
\end{aligned}
$$

that can be solved using the technic used in the invers kinematics of the two-link robotic manipulator, which yields

$$
\begin{aligned}
& q_{1}=\operatorname{atan} 2(y, x-b)-\operatorname{atan} 2\left(b S_{2}, 2 b+b C_{2}\right) \\
& q_{2}=\operatorname{atan} 2\left(S_{2}, C_{2}\right)
\end{aligned}
$$


where $C_{2}=\left((x-b)^{2}+y^{2}-5 b^{2}\right) / 4 b^{2}, S_{2}= \pm \sqrt{1-C_{2}^{2}}$. It is worth mentioning that two symmetrical solutions are possible here and both of them may be feasible, i.e. belong to the geometric limits described by (27). Then, for each segment the torque generated by the elastic virtual spring can be computed using (12), which for this mechanism is rewritten as

$$
M\left(q_{i}\right)=2 k\left[\left(b^{2}-a^{2}\right) \sin q_{i}-b L^{o} \sin \frac{q_{i}}{2}\right], i=1,2
$$
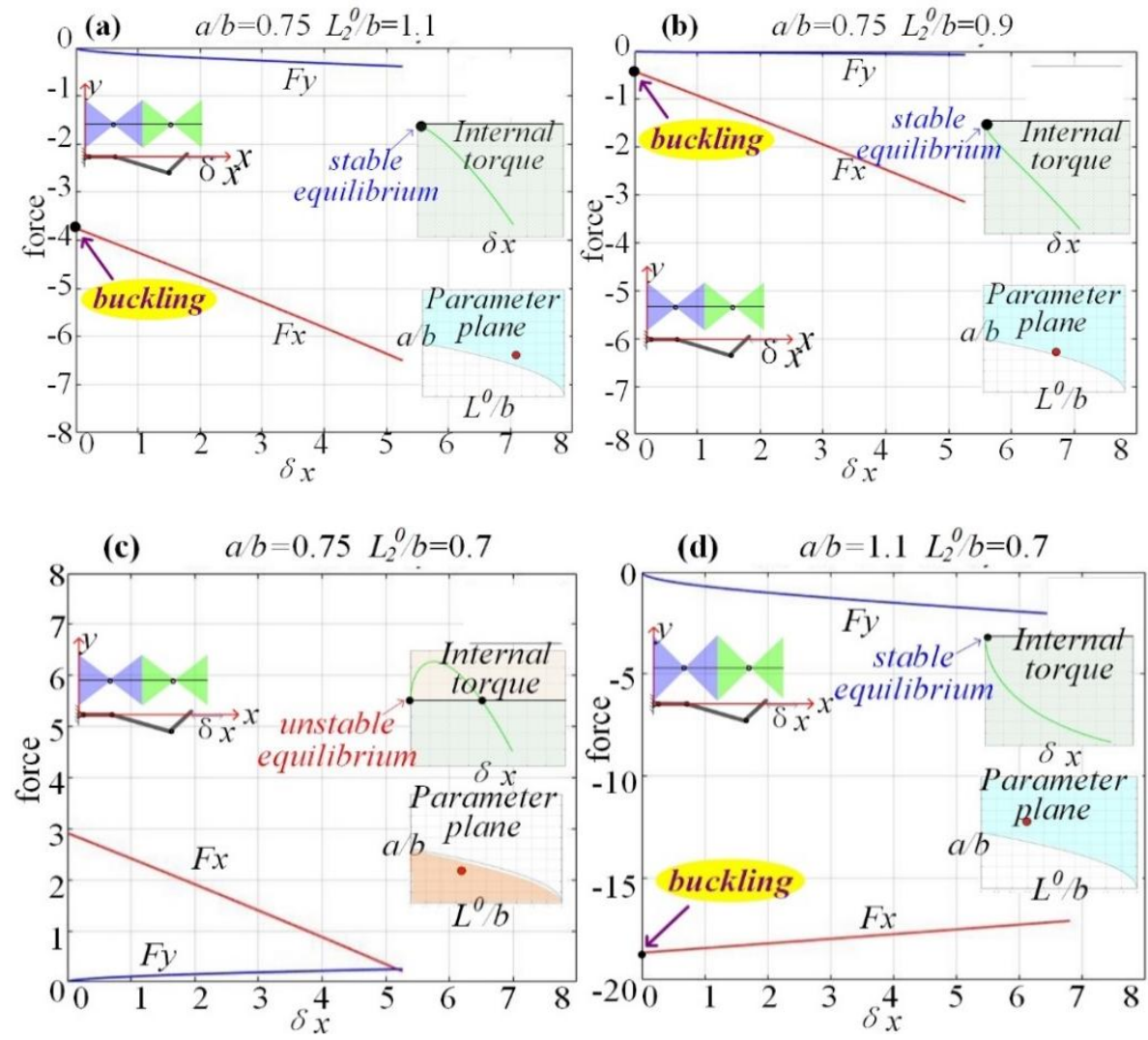

Fig. 10. Force-deflection relations $F_{x}\left(\delta_{x}, \delta_{y}\right), F_{y}\left(\delta_{x}, \delta_{y}\right)$ corresponding to unloaded straight configuration for different combinations of geometric parameters $a, b, L^{o}$

This allows us to obtain the desired equilibrium equation, which relates the virtual spring torques $M\left(q_{i}\right)$ and the external force $\left(F_{x}, F_{y}\right)^{T}$ in the following form

$$
\left[\begin{array}{l}
M\left(q_{1}\right) \\
M\left(q_{2}\right)
\end{array}\right]+J_{q}^{T}\left(\begin{array}{l}
F_{x} \\
F_{y}
\end{array}\right)=0
$$


where $J_{q}$ is the Jacobian matrix, which is derived from the geometry model and is written as follows

$$
J_{q}=\left[\begin{array}{cc}
-2 b S_{1}-b S_{1,2} & -b S_{1,2} \\
2 b C_{1}+b C_{1,2} & b C_{1,2}
\end{array}\right] ; \operatorname{det}\left(J_{q}\right)=2 b^{2} S_{2}
$$

and $C_{1}=\cos q_{1}, S_{1}=\sin q_{1}, C_{12}=\cos \left(q_{1}+q_{2}\right), S_{12}=\sin \left(q_{1}+q_{2}\right)$. After substitution of the virtual torques $M\left(q_{i}\right)$ in the above equilibrium equation, we can find the desired external force corresponding to the end-effector displacement $\left(\delta_{x}, \delta_{y}\right)$ expressed via the angles $\left(q_{1}, q_{2}\right)$

$$
\left(\begin{array}{l}
F_{x} \\
F_{y}
\end{array}\right)=2 k J_{q}^{-T}\left[\begin{array}{ll}
-\sin q_{1} & \sin \left(q_{1} / 2\right) \\
-\sin q_{2} & \sin \left(q_{2} / 2\right)
\end{array}\right]\left[\begin{array}{c}
b^{2}-a^{2} \\
b L^{0}
\end{array}\right],\left(q_{2} \neq 0\right)
$$

allowing us to obtain the desired force-deflection relation in the neighborhood of the straight configuration where the Jacobian is invertible. Relevant force-deflection curves for different combinations of the mechanism parameters are presented in Fig. 10.

Force $|\mathrm{Fx}|$

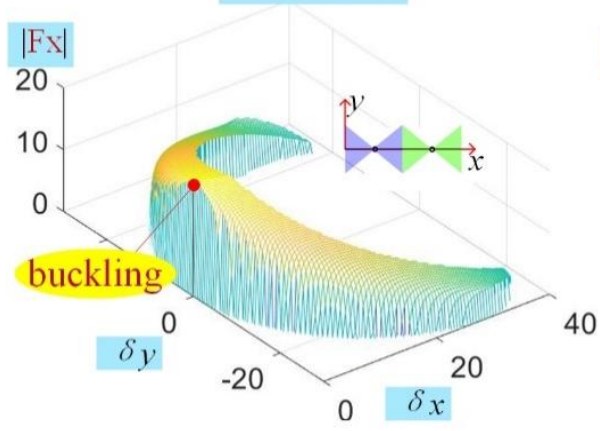

\section{Force $|\mathrm{Fy}|$}

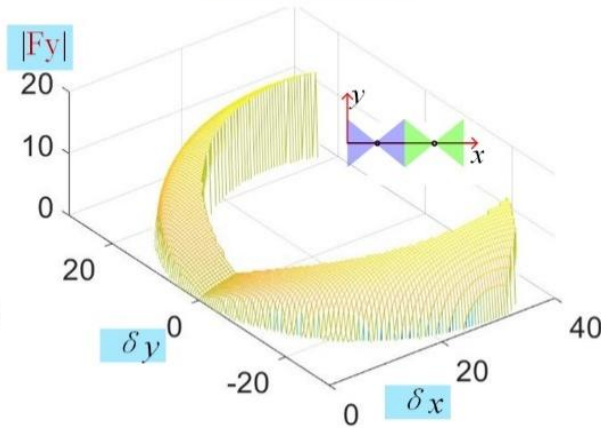

Fig. 11. Force-deflection relations $F_{x}\left(\delta_{x}, \delta_{y}\right), F_{y}\left(\delta_{x}, \delta_{y}\right)$ corresponding to unloaded straight configuration with parameters $a / b=1.1, L^{\mathrm{o}} / b=0.7$.

As follows from the force-deflection curves presented in Fig. 7, the mechanism stiffness behavior for the considered configuration possesses some particularities. In particular, at the beginning the mechanism resistance in the $x$-direction is infinite (corresponding curves do not go through the zero point). Further, when the loading is increasing, the mechanism resistance behavior is quasi-linear. Hence, the buckling phenomenon is observed when the external force increases gradually and the mechanism configuration angles suddenly change from zero to non-zero values. To find the 
critical force for the buckling, let us compute the limits of $\left(F_{x}, F_{y}\right)$ while $(\delta x, \delta y) \rightarrow(0,0)$.

As follows from the mechanism geometry, which include a triangle with edges length of $b$ and $2 b$, if the first angle $q_{1}=\varepsilon$ is small enough, the second angle can be approximately expressed as $q_{2} \approx-3 \varepsilon$. The later allows us to write the Jacobian in the following form

$$
J_{q} \approx\left[\begin{array}{cc}
0 & 2 b \varepsilon \\
3 b & b
\end{array}\right]
$$

and rewrite equation (34) as

$$
\left(\begin{array}{l}
F_{x} \\
F_{y}
\end{array}\right) \approx 2 k \cdot\left[\begin{array}{cc}
0 & 2 b \varepsilon \\
3 b & b
\end{array}\right]^{-T}\left[\begin{array}{cc}
-\varepsilon & \varepsilon / 2 \\
3 \varepsilon & -3 \varepsilon / 2
\end{array}\right]\left[\begin{array}{c}
b^{2}-a^{2} \\
b L^{0}
\end{array}\right]
$$

that gives us the desired critical forces in the $x$ - and $y$-directions

$$
\begin{aligned}
& F_{x}^{0}=\lim _{\varepsilon \rightarrow 0} F_{x}(\varepsilon)=5 k\left[2\left(b^{2}-a^{2}\right)-b L^{o}\right] / 3 b \\
& F_{y}^{0}=\lim _{\varepsilon \rightarrow 0} F_{y}(\varepsilon)=0
\end{aligned}
$$

It should also be mentioned that the buckling phenomenon can be observed if and only if $L^{0} / b>2\left(1-a^{2} / b^{2}\right)$, which in the previous section was considered as the boundary condition separating the monotonic and non-monotonic areas in Fig. 3 ( see in Eq. 15 ). In this case, the unloaded straight configuration is stable and it resists to the external loading if $F_{x}<F_{x}^{0}$. In contrast, if the geometry satisfies the opposite condition: $L^{0} / b<2\left(1-a^{2} / b^{2}\right)$ as shown in Fig. 10c, the unloaded configuration is unstable and the mechanism suddenly jumps from the initial position to slightly different stable equilibriums (even without external loading), which can be treated as the "jumping" phenomenon. The properties of the force-diflection relations $F_{x}\left(\delta_{x}, \delta_{y}\right)$ and $F_{y}\left(\delta_{x}, \delta_{y}\right)$ can be also estimated from the 3D-plots presented in Fig. 11, where the buckling corresponds to the discontinuty at the point $\left(\delta_{x}, \delta_{y}\right)=(0,0)$.

\subsection{Stiffness Analysis of Non-Straight Configurations}

To evaluate the mechanism stiffness for the general case, let us assume that the initial unloaded configuration is non-straight, i.e. $q_{2} \neq 0$, which guaranties that the kinematic Jacobian is non-singular and can be inverted. This allows us to rewrite the equilibrium equation in the following way 


$$
\left(\begin{array}{l}
F_{x} \\
F_{y}
\end{array}\right)=-J_{q}^{-T} K_{q} J_{q}^{-1}\left(\begin{array}{l}
\delta x \\
\delta y
\end{array}\right)
$$

where the diagonal matrix $K_{q}=\operatorname{diag}\left(K_{q 1}, K_{q 2}\right)$ is composed of the stiffness coefficients of the virtual joints $K_{q i}=d M\left(q_{i}\right) / d q_{i}$ described by (20). This equation also allows us to express the mechanism $2 \times 2$ stiffness matrix explicitly in the following form

$$
K_{F}=J_{q}^{-T} K_{q} J_{q}^{-1}
$$

where

$$
K_{F}=\left[\begin{array}{ll}
k_{x x} & k_{x y} \\
k_{y x} & k_{y y}
\end{array}\right]_{2 \times 2}
$$

is the symmetrical positive definite matrix composed of scalar stiffness coefficients $k_{x x}, k_{y y}$ for the directions $x$ and $y$, as well as a mixed stiffness coefficient $k_{x y}$.
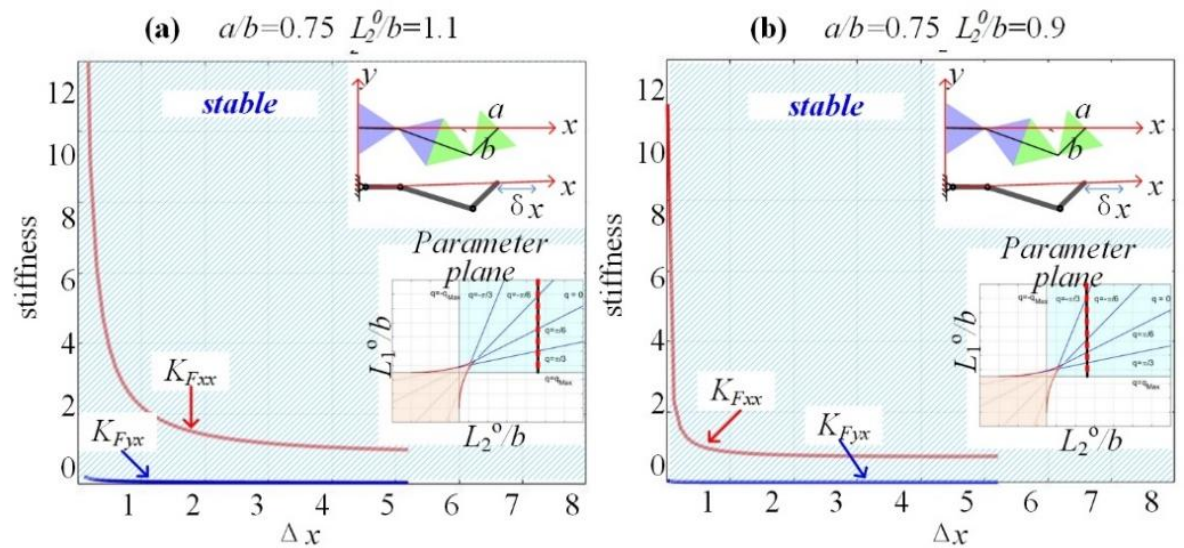
(c) $a b=0.75 \quad L_{2}^{0} b=0.7$

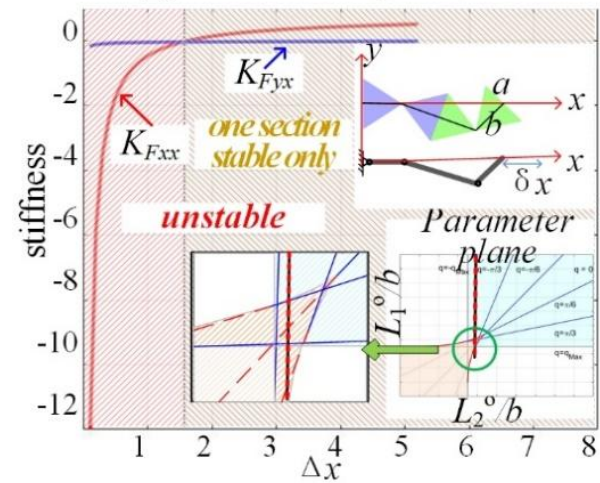

(d) $a / b=1.1 \quad L_{2}^{0} b=0.7$

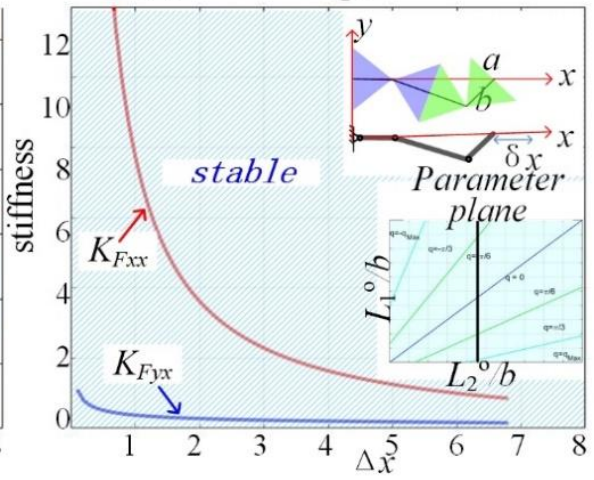

Fig. 12. Sensitivity of the stiffness coefficients of the two-segment mechanism with respect to initial unloaded configuration for different geometric parameters.
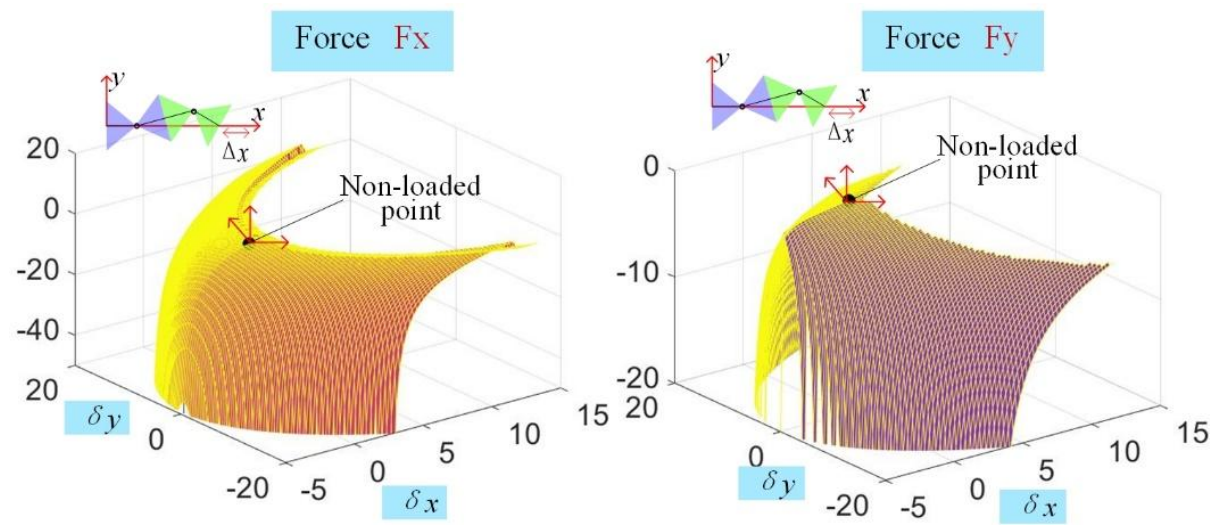

Fig. 13. Force-deflection relations $F_{x}\left(\delta_{x}, \delta_{y}\right), F_{y}\left(\delta_{x}, \delta_{y}\right)$ corresponding to unloaded nonstraight configuration $\left(\Delta_{x}, \Delta_{y}\right)=(5,0)$ with geometric parameters $a / b=1.1, L^{\mathrm{o}} / b=0.7$.

Let us investigate now the sensitivity of the above stiffness coefficients with respect to the control inputs $L_{i 1}^{0}$ and $L_{i 2}^{0}$, assuming that both segments of the mechanism are controlled by single inputs, i.e. $L_{11}^{0}=v a r_{1}, L_{21}^{0}=v a r_{2}$ and $L_{12}^{0}=L_{22}^{0}=$ const , which insure the desired unloaded end-point location $(x, y)=(4 b-\Delta x, 0)$. Corresponding computation results for several case studies are presented in Fig. 12, they demonstrate that the stiffness of the two-segment mechanism is very sensitive to its initial unloaded configuration. In particular, the mechanism stiffness coefficients for the $x$-direction are essentially reducing while the displacement $\Delta x$ is increasing. It should be mentioned that, to have the stable equilibrium configuration, both two segments of the mechanism should satisfy the stability condition presented in the previous section. The latter is illustrated by Fig. 12c, where the right-hand side segment of the mecha- 
nism is stable ( $K_{q 2}<0$ ), while the left-hand side segment is in unstable configuration $\left(K_{q 1}>0\right)$. So the left-hand side segment moves until being stopped by the rotation angle constrain. This situation is also in accordance with the control inputs location on the parameter plane $\left(L_{1}^{0} / b, L_{2}^{0} / b\right)$ shown in Fig. 11c, which allows evaluate the segment stability in the unloaded configuration.

The stiffness properties of the two-segment mechanism for the non-straight initial configuration can be also estimated from 3D-plots of the force-diflection relations $F_{x}\left(\delta_{x}, \delta_{y}\right)$ and $F_{y}\left(\delta_{x}, \delta_{y}\right)$ presented in Fig. 13, which correspond to the non-loaded displacements $\left(\Delta_{x}, \Delta_{y}\right)=(5,0)$. As follows from this plots, the forces $F_{x}$ and $F_{y}$ are changing continuously with respect to the deflections $\left(\delta_{x}, \delta_{y}\right)$, and there is no the buckling phenomenon, that was detected for the straight initial configuration (see Fig. 11 in subsection 3.1).

Another way of evaluating the mechanism stiffness properties is based on the singular value decomposition (SVD) of the $2 \times 2$ stiffness matrix computed at the initial unloaded point. This approach allows us to estimate the max/min compliance of the mechanism via the stiffness matrix singular values [26]. Geometrically, it can be also described by the stiffness ellipse that is produced by the linear mapping (38) of the unit circle $\delta_{x}^{2}+\delta_{y}^{2}=1$ in the deflection space into the force space ( $F_{x}, F_{y}$ ). It can be proved that the lengths of the major and minor semi-axes of this ellipse are equal respectively to the maximum and minimum singular values of the stiffness matrix. Example of the stiffness ellipses for the case $a / b=1.1, L^{\circ} / b=0.7$ and the evaluation though out the workspace is presented in Fig. 14. As follows from this figure, the mechanism resistance to the external force in the longitudinal direction is much higher than in the transverse one. Also, the mechanism longitudinal compliance increases while the initial configuration becomes closer to the straight one.

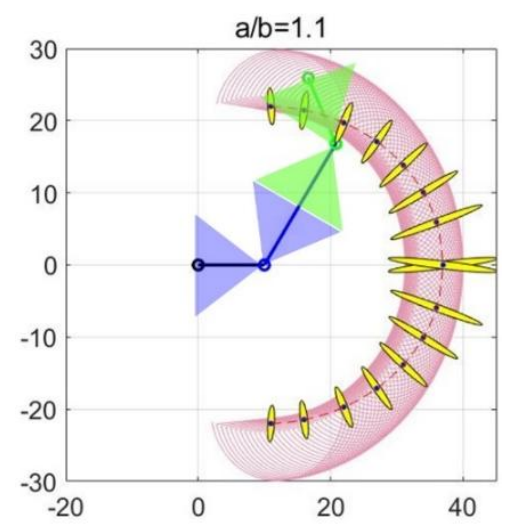

Fig. 14. Stiffness ellipses of the two-segment mechanism and the evaluation through the workspace. 


\subsection{Controlling Mechanism Configurations}

To achieve the desired end-point position $(x, y)$, the two-segment mechanism must be controlled by two pairs of the control inputs $\left(L_{11}^{0}, L_{12}^{0}\right)$ and $\left(L_{21}^{0}, L_{22}^{0}\right)$. To simplify the mechanism control, let us apply the asymmetrical approach proposed in subsection 1.3 allowing to use only two control variables $\left(\Delta_{1}, \Delta_{2}\right)$ producing four physical control inputs: $L_{11}^{0}=L^{0}-\Delta_{1}, L_{12}^{0}=L^{0}+\Delta_{1}$ for the first segment, and $L_{21}^{0}=L^{0}-\Delta_{2}$, $L_{22}^{0}=L^{0}+\Delta_{2}$ for the second one. Since the considered two-segment mechanism is non-redundant, the values of the control variables corresponding to the desired endpoint position can be easily computed using the above presented expressions for the two-link manipulator inverse kinematics (30) and one-segment mechanism control law (26). The latter yields the following algorithm for controlling law of the twosegment mechanism:

1. Using expressions (30), compute the configuration angles $q_{1}(x, y)$ and $q_{2}(x, y)$ corresponding to the desired end-point position $(x, y)$.

2. Using expression (26), compute the control inputs $\Delta_{1}\left(q_{1}\right)$ and $\Delta_{2}\left(q_{2}\right)$ for the first and second segments corresponding to the configuration angles $\left(q_{1}, q_{2}\right)$.

An example of computing based on the above algorithm is presented in Fig. 15, where the mechanism parameters $a / b=1.1, L^{0} / b=0.7$ were chosen to ensure the mechanism stability in the unloaded mode $M_{e x t}=0$ (see subsection 2.3 ).
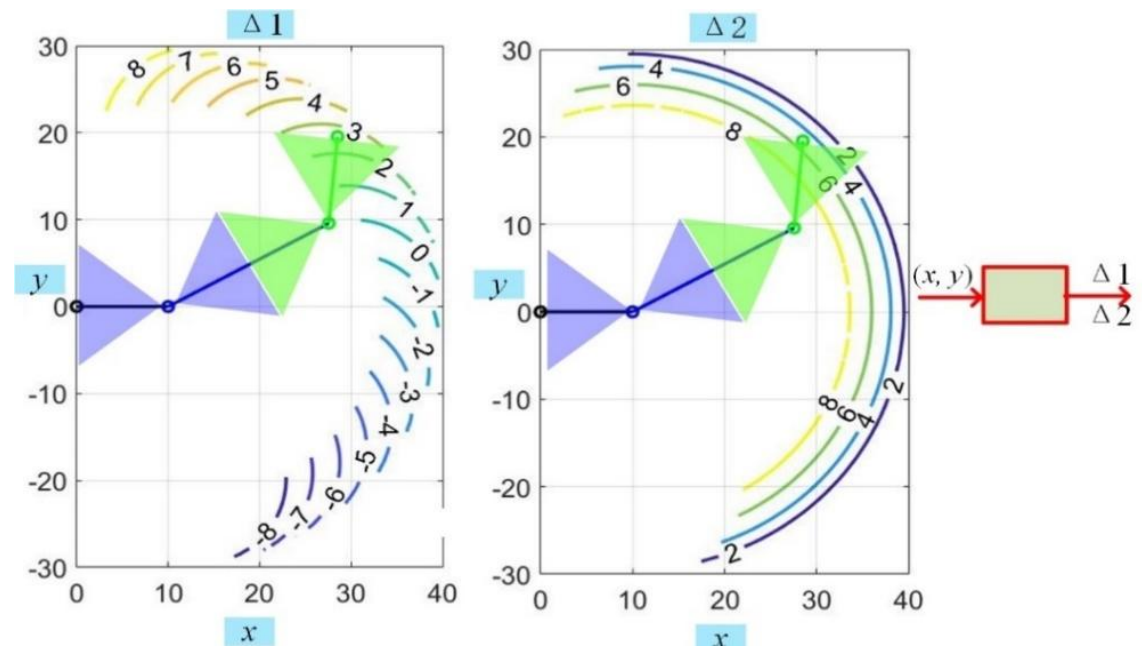

Fig. 15. Relations between the required control inputs $\Delta_{1}, \Delta_{2}$ and the desired end-point position $(x, y)$ for the two-segment mechanism with geometric parameters $a / b=1.1, L^{\mathrm{o}} / b=0.7$ (unloaded case $\left.F_{x}=F_{y}=0\right)$. 

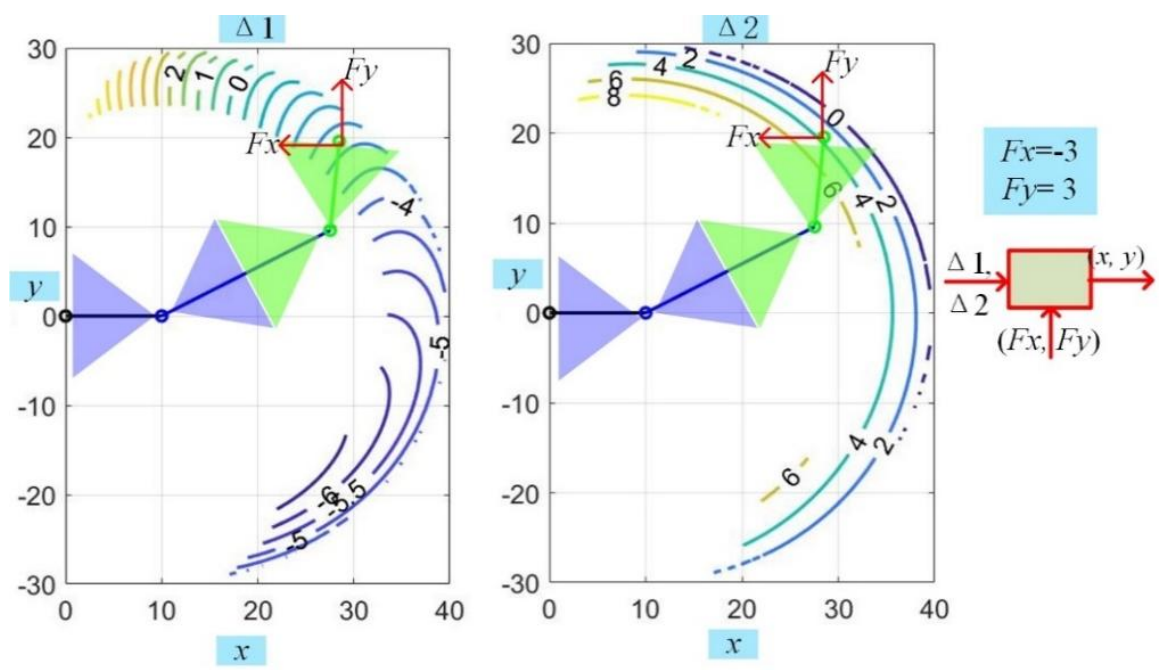

Fig. 16. Relations between the control input $\Delta$ and the desired end-point position $(x, y)$ with geometric parameters $a / b=1.1, L^{\mathrm{o}} / b=0.7$ (unloaded case $F_{x}=-3, F_{y}=3$ ).

In more general case when the external forces $\left(F_{x}, F_{y}\right)$ are not equal to zero, the control inputs $\left(\Delta_{1}, \Delta_{2}\right)$ should be computed using equation (28) that includes the virtual joint torques $\left(M_{1}, M_{2}\right)$ ensuring the mechanism equilibrium.

These torques can be obtained from the static equilibrium (32). Corresponding algorithm allowing to find the control inputs $\left(\Delta_{1}, \Delta_{2}\right)$ for the desired end-point position $(x, y)$ for the loaded case as presented below:

1. Using expressions (30), compute the configuration angles $q_{1}(x, y)$ and $q_{2}(x, y)$ corresponding to the desired end-point position $(x, y)$.

2. Using expression (32), compute the joint torques $\left(M_{q 1}, M_{q 2}\right)$ from the external force $\left(F_{x}, F_{y}\right)$.

3. Using expression (28), compute the control inputs $\Delta_{1}\left(q_{1}, M_{1}\right)$ and $\Delta_{2}\left(q_{2}, M_{2}\right)$ for the first and second segments corresponding to the configuration angles $\left(q_{1}, q_{2}\right)$ and the joint torques $\left(M_{q 1}, M_{q 2}\right)$.

An example of computing based on the above algorithm is presented in Fig. 16, where the geometric parameters are similar to the previous figure.

\section{Conclusion}

The paper presents some new results on the equilibrium configurations analysis of a new type of compliant tensegrity mechanism as mentioned in our previous paper [24], which is composed of rigid triangles connected by passive joints. In contrast to con- 
ventional cable driven mechanisms, here there are two length-controllable elastic edges that can generate internal preloading. So, the mechanism can change its equilibrium configuration by adjusting the control inputs length. Such design is very promising and convenient for constructing a multi-section serial structures with high flexibility, which are needed in many modern robotic applications.

For a single segment mechanism, the main attention was paid to a symmetrical structure composed of similar triangles. In particular, the case of equal control inputs was investigated in detail and analytical condition of equilibrium stability was obtained, which allows users to select the suitable control inputs ensuring the mechanism controllability. The control algorithm for such mechanism based on the above stable condition analysis was presented also, which was approved efficient through simulation for defining different control inputs to get the desired stable equilibrium configurations for this type of mechanism. The relation between the external torque and the deflection was also obtained allowing to find loaded equilibriums. It was proved that depending on parameters combinations, the actuation can lead to either the desired mechanism configuration (corresponding to a stable equilibrium) or undesired configuration corresponding to shifted stable equilibrium or joint limits. Besides, similar analysis has been done for the case of non-equal control inputs, and equivalent serial structure was proposed where the passive joint was replaced by a virtual actuated joint with variable stiffness. It was shown that, to achieve the desired stable equilibrium configuration, the control inputs should not only satisfy the condition of stability, but also been located inside the feasible regions. For a two segment mechanism, the buckling phenomenon where detected occurring when the mechanism initial configuration is straight. However, in contrast to the traditional buckling, such phenomenon does not lead to unstable configuration. The 3D plots of the forcedeflection relation curves were obtained through simulation for both the initial straight and non-straight configurations, which demonstrated the continuous change of the mechanism stiffness after buckling.

In future, these results will be used for the stiffness analysis of multi-segment mechanisms that may demonstrate unusual behavior under static load and suddenly change its configuration, if the control inputs do not satisfy the stability condition.

\section{Acknowledgements}

This work was supported by the China Scholarship Council ( No. 201801810036 ).

\section{References}

1. Frecker, M. I., Ananthasuresh, G. K., Nishiwaki, S., Kikuchi, N., \& Kota, S. Topological Synthesis of Compliant Mechanisms Using Multi-Criteria Optimization. Journal of Mechanical Design, 119(2), 238-245 (1997).

2. Albu-Schaffer, A., Eiberger, O., Grebenstein, M., Haddadin, S., Ott, C., Wimbock, T., Wolf, S., \& Hirzinger, G. Soft robotics. IEEE Robotics Automation Magazine, 15(3), 2030 (2008). 
3. Wang, M. Y., \& Chen, S. Compliant mechanism optimization: analysis and design with intrinsic characteristic stiffness. Mechanics Based Design of Structures and Machines, 37(2), 183-200 (2009).

4. Howell, L. L. Compliant mechanisms. In 21st century kinematics (pp. 189-216). Springer, London. (2013).

5. Pan, Y., Wang, H., Li, X., \& Yu, H. Adaptive Command-Filtered Backstepping Control of Robot Arms With Compliant Actuators. IEEE Transactions on Control Systems Technology, 26(3), 1149-1156, (2018).

6. Leidner, D. S. Cognitive Reasoning for Compliant Robot Manipulation (Vol. 127). Springer International Publishing, (2019).

7. Robinson, Graham, and J. Bruce C. Davies. "Continuum robots-a state of the art." Proceedings 1999 IEEE international conference on robotics and automation (Cat. No. 99CH36288C). Vol. 4. IEEE, (1999).

8. Chirikjian, G. S., \& Burdick, J. W. Kinematically optimal hyper-redundant manipulator configurations. IEEE Transactions on Robotics and Automation, 11(6), 794-806 (1995).

9. Yang, J., Pitarch, E. P., Potratz, J., Beck, S., \& Abdel-Malek, K. Synthesis and analysis of a flexible elephant trunk robot. Advanced Robotics, 20(6), 631-659, (2006).

10. Chirikjian, G. S., \& Burdick, J. W. A modal approach to hyper-redundant manipulator kinematics. IEEE Transactions on Robotics and Automation, 10(3), 343-354, (1994).

11. Anderson, V. C., \& Horn, R. C. U.S. Patent No. 3,497,083. Washington, DC: U.S. Patent and Trademark Office, (1970).

12. Gravagne, I. A., \& Walker, I. D. Kinematic transformations for remotely-actuated planar continuum robots. Proceedings 2000 ICRA. Millennium Conference. IEEE International Conference on Robotics and Automation. Symposia Proceedings (Cat. No.00CH37065), 1, 19-26 vol.1, (2000).

13. Cieślak, R., \& Morecki, A. Elephant trunk type elastic manipulator-A tool for bulk and liquid materials transportation. Robotica, 17(1), 11-16, (1999).

14. Rolf, M., \& Steil, J. J. Constant curvature continuum kinematics as fast approximate model for the Bionic Handling Assistant. 2012 IEEE/RSJ International Conference on Intelligent Robots and Systems (pp. 3440-3446). IEEE(2012).

15. Yang, Y., \& Zhang, W. An elephant-trunk manipulator with twisting flexional rods. 2015 IEEE International Conference on Robotics and Biomimetics (ROBIO) (pp. 13-18). $\operatorname{IEEE}(2015)$.

16. Skelton, R. E., \& de Oliveira, M. C. Tensegrity systems (Vol. 1). New York: Springer(2009).

17. Moored, K. W., Kemp, T. H., Houle, N. E., \& Bart-Smith, H. Analytical predictions, optimization, and design of a tensegrity-based artificial pectoral fin. International Journal of Solids and Structures, 48(22-23), 3142-3159 (2011).

18. Duffy, J., Rooney, J., Knight, B., \& Crane III, C. D. A review of a family of self-deploying tensegrity structures with elastic ties. Shock and Vibration Digest, 32(2), 100-106 (2000).

19. Arsenault, M., \& Gosselin, C. M. Kinematic, static and dynamic analysis of a planar 2DOF tensegrity mechanism. Mechanism and Machine Theory, 41(9), 1072-1089 (2006).

20. Furet, M., Lettl, M., \& Wenger, P. Kinematic analysis of planar tensegrity 2-X manipulators. International Symposium on Advances in Robot Kinematics (pp. 153-160). Springer, Cham (2018).

21. Furet, M., \& Wenger, P. Workspace and cuspidality analysis of a 2-X planar manipulator. IFToMM Symposium on Mechanism Design for Robotics (pp. 110-117). Springer, Cham (2018). 
22. Wenger, P., \& Chablat, D. Kinetostatic analysis and solution classification of a planar tensegrity mechanism. Computational Kinematics (pp. 422-431). Springer, Cham (2018).

23. Moon, Y., Crane, C. D., \& Roberts, R. G. Position and force analysis of a planar tensegrity-based compliant mechanism. Journal of Mechanisms and Robotics, 4(1) (2012).

24. Zhao, W.; Pashkevich, A.; Klimchik, A. and Chablat, D. Stiffness Analysis of a New Tensegrity Mechanism based on Planar Dual-triangles. Proceedings of the 17th International Conference on Informatics in Control, Automation and Robotics - Volume 1: ICINCO, 402-411. (2020)

25. Jones, R. M. Buckling of bars, plates, and shells. Bull Ridge Corporation (2006).

26. Yuan, J. Local SVD inverse of robot Jacobians. Robotica, (2001). 